\title{
Paternal care and the cost of polygyny in the green dart-poison frog
}

\author{
Kyle Summers \\ Division of Amphibians and Reptiles, Museum of Zoology, University of Michigan, Ann Arbor, MI 48109, USA, and \\ Smithsonian Tropical Research Institute, P.O. Box 2072, Balboa, Republic of Panama \\ Received January 8, 1990 / Accepted July 5, 1990
}

Summary. In species with male parental care, polygyny may reduce the parental effort provided by a male, and hence impose a cost on the fitness of his mates, because of decreased growth, survival or health of offspring. I examined a cost of polygyny in the green dart-poison frog, Dendrobates auratus, a species with male parental care in which both male polygyny and mate guarding by females occurs (Summers 1989). All D. auratus males seen carrying tadpoles in a marked area were followed and the pools where they deposited their tadpoles were recorded. Males frequently deposited more than one tadpole in the same pool (in $25 \%$ of the observed depositions a male deposited a tadpole in a pool where he had previously deposited at least one other tadpole). Experiments involving manipulation of tadpole densities in pools typically utilized by $D$. auratus (calabash husks and treeholes) showed that increasing tadpole number had a strong negative effect on both growth rate and survivorship, indicating that polygyny can impose a cost on the fitness of a male's offspring. Hence, females do face a potential cost, in terms of reduced offspring growth and survivorship, when their mates mate polygynously and care for the offspring of other females.

\section{Introduction}

In the animal kingdom, the amount of parental investment an individual's mate will put into it's gametes and/ or offspring may be unpredictable. Because of the initial disparity in gamete size and mobility that defines the sexes, this type of uncertainty (which I will refer to as uncertainty of parental investment), as well as the degree of confidence an individual has that a particular offspring is its own (confidence of parentage), usually derive from uncertainty about whether the female's eggs have been fertilized by the male's sperm for males, while for females they usually derive from uncertainty concerning whether or not her mate will care for the offspring of other females. Unlike relative parental invest- ment (Trivers 1972), uncertainty of parental investment selects for aggressive mate guarding and selectivity in the same sex (Summers 1989). Effort expended on this type of aggression should be considered mating effort, even though it may occur after mating takes place, because it's purpose is to extract parental investment from a mate, and it does no increase the rate of population growth (and is therefore part of the cost of sex, Alexander and Borgia 1979).

Uncertainty of parental investment will only be important if members of one sex suffer a cost (in terms of reduced parental investment in their offspring) when their mates mate polygamously. This type of data abounds for males (e.g Thornhill and Alcock 1983), but data showing that females suffer a cost through reduction in the quality or quantity of paternal care their offspring receive when their mates mate polygynously are rare (e.g. Yasukawa and Searcy 1982). This paper presents evidence that female green dart-poison frogs, Dendrobates auratus, potentially suffer a cost, in terms of reduced offspring growth rate and survivorship, when their mates are polygynous and care for the offspring of other females.

Dendrobates auratus, the green dart-poison frog, is a member of the genus Dendrobates, a diurnal, terrestrial group of neotropical frogs known as dart-poison frogs because glands in their skin produce neurotoxic alkaloids. Previous research on this species has demonstrated a high degree of male parental care, and indicated that some females attempt to guard their mates from other females for long periods (at least several months), through aggression and active courtship (Summers 1989). Paternal care involves periodic attendance of the eggs until they become tadpoles, location of suitable pools for tadpoles, and transportation of tadpoles to pools.

In the summer of 1988 , I attempted to determine if females suffer a cost when their mates mate with other females and care for their offspring. Because of the complexity of parental care in this species, there are several possible ways in which a male's polygyny might nega- 
tively affect the average fitness of his offspring. Direct costs to eggs and tadpoles might result from reduced frequency and duration of egg attendance (e.g from increased dessication). The amount of time males spend searching for a deposition site for each tadpole could decrease as the number of clutches a male cares for increases. It is also possible that polygynous males deposit more than one tadpole in a pool more frequently as their degree of polygyny increases. Previous observations (Summers, unpublished data) suggest that males tend to deposit the tadpoles from a single clutch in different pools. However, it is possible that a male will tend to deposit more than one tadpole in a pool as his mating success, and hence the number of clutches he cares for, increases.

In this study, I chose to investigate the effect of the number of tadpoles deposited in a pool on the fitness of the tadpoles in the pool, as measured by growth rate and survivorship. If individual males deposit more than one tadpole in the same pool, then females may suffer a cost from increased mortality or decreased growth rate of the tadpoles (males suffer this cost as well, but from their perspective it may be mitigated by higher overall offspring production). This cost was investigated because it appeared likely that males were limited in their ability to locate tadpole deposition sites, and because it seemed the most tractable cost to investigate empirically.

To determine if this is a potential cost to females, one needs to answer two questions. First, do individual males carry multiple tadpoles to 1 pool, and second, does increasing the number of tadpoles in a pool affect their growth or survivorship? In this study, I first observed tadpole deposition by marked males. Second, I measured the growth rates and survivorship of tadpoles in experimentally manipulated pools.

\section{Methods}

The research was carried out on Taboga Island, in the Republic of Panama. For the study of tadpole deposition patterns, all males within a $12 \times 12 \mathrm{~m}$ area were marked by drawing their dorsal color patterns on ID cards and by toe-clipping. The area was then observed continuously from 6 am to $6 \mathrm{pm}, 6$ days a week, from May 4th to June 20th. All males from the area observed carrying a tadpole were followed, and where they deposited their tadpole was recorded. Frogs carrying tadpoles to treehole pools were followed using climbing rope, a tree trimming harness, and rope climbing devices (jumars).

For the study of tadpole ecology, a series of pools were created using calabash (Crescentia) husks filled with 9.5 grams of detritus and leaf litter. The first of these pools were started in early June and pools were started periodically until early August. Such pools occur naturally on Taboga and are utilized by $D$. auratus as tadpole deposition sites (personal observations). The main set of treatments involved varying the number of tadpoles placed in a pool at the start of the experiment. Pools were started with 1 tadpole per pool $(N=6), 2$ tadpoles per pool $(N=9), 4$ tadpoles per pool $(N=12)$, 8 tadpoles per pool $(N=16)$, and 16 tadpoles per pool $(N=7)$. Three measures of size (total length, head width and snout-vent length) were taken on each tadpole when each pool was started, weekly for 4 weeks, at 6 weeks, and finally at metamorphosis. Because of time constraints, weight, size and/or mortality were not measured in each pool each week, and sample sizes consequently varied between weeks and between measures (e.g. weight and mortality). The weight of all tadpoles in a pool was measured at the same time, using an Ohaus portable field scale. Survivorship was determined by counting the tadpoles in the pool each week (pools were checked daily for tadpoles nearing metamorphosis, which were easy to recognize, in order to insure that no tadpoles metamorphosed and left without being seen). The sample size of metamorphs is reduced because tadpoles from pools begun late in the study did not metamorphose by the end of the study.

Although I attempted to utilize husks of similar size, and placed marks inside the husks to indicate the water level at which the volume equaled $280 \mathrm{ml}$, there was some differential evaporation, so the volumes of the pools were only approximately equivalent. Tadpoles were obtained by placing plastic cups in the field and collecting the tadpoles deposited in them every $24 \mathrm{~h}$. Hence, all the tadpoles in a pool started at similar sizes and ages. Data from 5 pools with one tadpole each were discarded because the detritus used in these pools (which were started simultaneously) was later found to have been contaminated with cat urine. Leaving these pools in the analysis reduced the $\mathrm{R}^{2}$ for the regressions slightly, but the level of significance remained identical to three decimal places for both regressions $(P=0.000)$. The tadpole experiments were run from June 10th to September 10th, 1988.

I also ran an experiment on the effect of tadpole number in a series of pools in treeholes, another type of pool commonly used by $D$. auratus for tadpole deposition, by removing all the tadpoles in a treehole, then depositing a previously weighed and measured cohort of one day old tadpoles obtained from the cups. Two different sized (i.e. number of tadpoles) cohorts were placed in the pool sequentially (high and low, the actual numbers depending on the size of the pool and the number of tadpoles available). Each cohort was allowed to grow for 2 or 3 weeks (depending on the pool), and then the tadpoles left were removed and weighed and measured. Plastic screening was used to prevent new tadpoles from being deposited once the experiment was in progress. The mesh was wide enough to allow mosquitos to deposit eggs (a source of food for the tadpoles). Due to a variety of logistical difficulties, I was only able to get data from 8 treehole pools. In 4 of these pools the high tadpole number cohort was placed in the pool first, while the low tadpole number cohort was placed in the pool first in the other 4 .

In addition to the experiments involving number of tadpoles per pool, 2 other treatments were given in separate experiments; 1) the effect of size was examined by pairing 2 tadpoles of disparate sizes (one small: between 6 and $7 \mathrm{~mm}$ snout-vent length, and one large: between 8 and $15 \mathrm{~mm}$ SVL) in calabashes and measuring growth and survivorship. 2) the effect of food availability was examined by providing superabundant hi-protein goldfish food (i.e. enough food that there was uneaten food visible in the pool each day) to a series of tadpole number treatments in plastic cups.

The data were analyzed on a microcomputer with SYSTAT (Wilkinson 1987). When parametric tests were employed, the residuals were examined to insure that the data met the requirements of normality and homogeneity of variances. Two-tailed tests were employed unless otherwise noted. In some cases, the data were transformed in order to provide a better fit to the assumptions of the parametric tests.

\section{Results}

\section{Tadpole deposition}

The results of the observations on tadpole carrying males are shown in Table 1. The top row gives the ID numbers of the males who were followed, and the first column gives the pools where males were seen depositing tadpoles; about $25 \%$ of the time males deposited a tad- 
Table 1. The number of observed tadpole depositions by 11 males in 12 pools

\begin{tabular}{|c|c|c|c|c|c|c|c|c|c|c|c|}
\hline \multirow[t]{2}{*}{ Pool \# } & \multicolumn{11}{|c|}{ Male \# } \\
\hline & 9 & 141 & 143 & 115 & 23 & 11 & 12 & 10 & 22 & 146 & 149 \\
\hline 1 & & & 2 & & & 1 & 1 & 1 & & & \\
\hline 2 & & & & & & 1 & & & & & \\
\hline 3 & 1 & & & & & 1 & 1 & & & & \\
\hline 4 & 1 & & & & & & & & & & \\
\hline 5 & & & & 3 & & & & & & 2 & \\
\hline 6 & & & & 1 & & & & & & & \\
\hline 7 & & & & & & & 1 & & & & \\
\hline 8 & & & 1 & & & & & 1 & & & \\
\hline 9 & 1 & & & & & & & & & & \\
\hline 10 & & 5 & & 1 & 2 & & & & 2 & & \\
\hline 11 & 1 & & & & & & & & & & \\
\hline 12 & 1 & & & & & & & & & & 1 \\
\hline
\end{tabular}

pole in a pool where they had previously deposited one or more tadpoles.

\section{Growth rate}

During the first 4 weeks of the study, there was a significant negative relationship between the average growth rate and the number of tadpoles placed in the calabash at the start of the experiment, as measured by weight

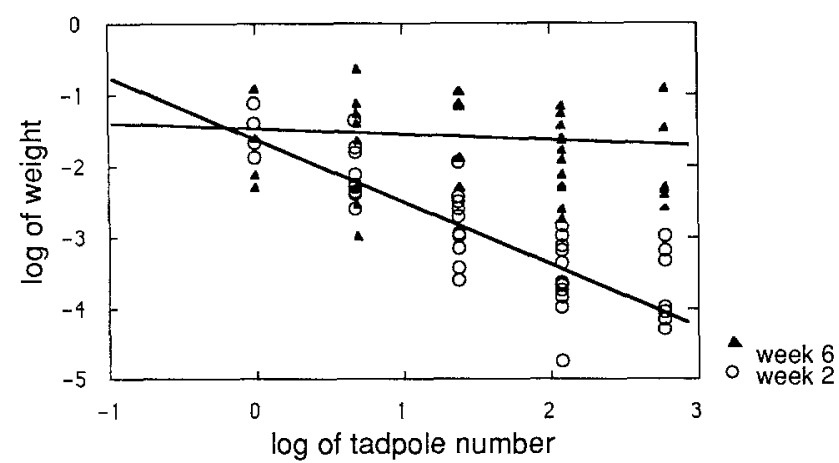

Fig. 1. Linear regressions of the log of average tadpole weight on the log of the number of tadpoles placed in the pool at the start of the experiment, at week 2 and at week 6

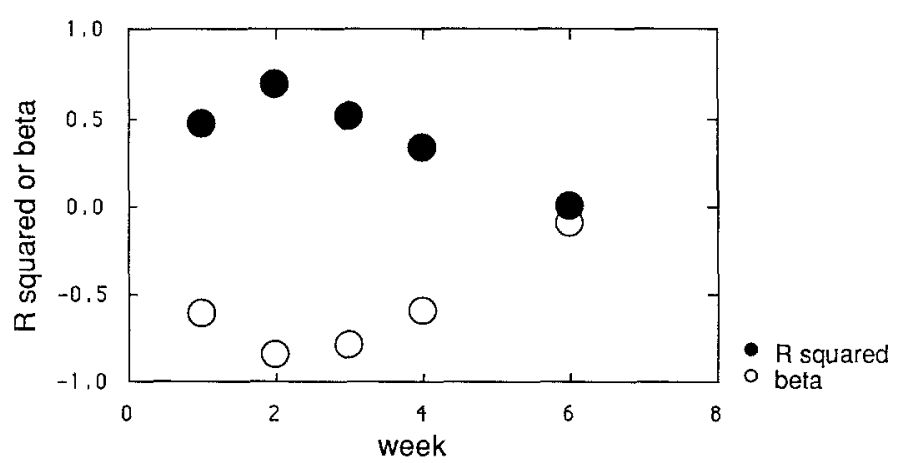

Fig. 2. Plot of the amount of variance explained by a linear regression of the log of average tadpole weight on the log of tadpole number ( $R$ squared), and the coefficient of the regression (beta), by week, from week 1 through week 6 and size. For example, there was a significant negative relationship between the $\log$ of weight and the $\log$ of tadpole number at week 2 (Fig. $1, R^{2}=0.689, N=46$, $P=0.000$ ). A significant negative relationship was also found between tadpole length, and the $\log$ of tadpole number $\left(R^{2}=0.642, N=45, P=0.000\right)$. By week six, however, the relationship between weight (and size) and number was no longer significant (Figure $1, R^{2}=0.000$, $N=34, P=0.407$, for weight). Most growth in the low tadpole number treatments occurred during the first few weeks, so the difference in the relationship between tadpole number and average tadpole weight apparently had to do with changes in the high tadpole number treatments (see below). The amount of variance explained by the regression of the $\log$ of weight on the $\log$ of tadpole number reaches a peak at week 2 , and declines to 0 by week 6 (Fig. 2).

\section{Mortality}

Overall mortality increased with time (Fig. 3). Average mortality was higher in the treatments with more tad-

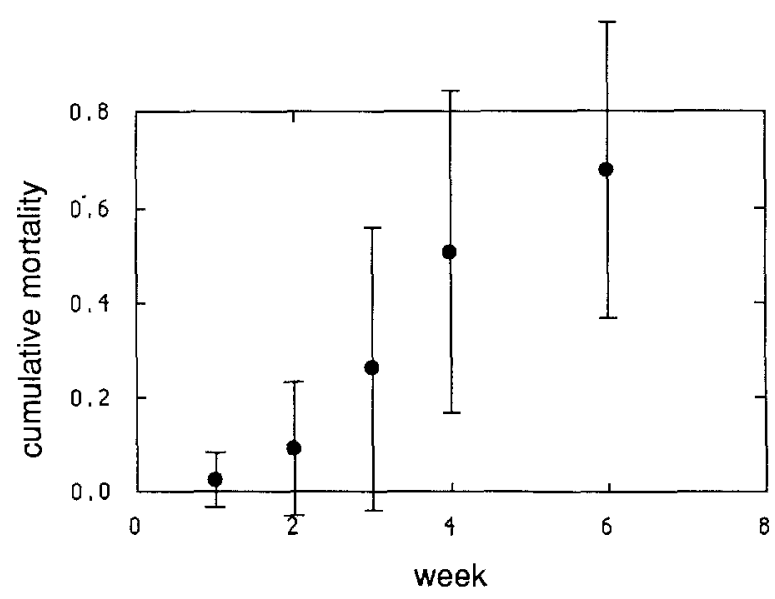

Fig. 3. Plot of average tadpole mortality by week, for week 1 through 6. Error bars show the standard deviation

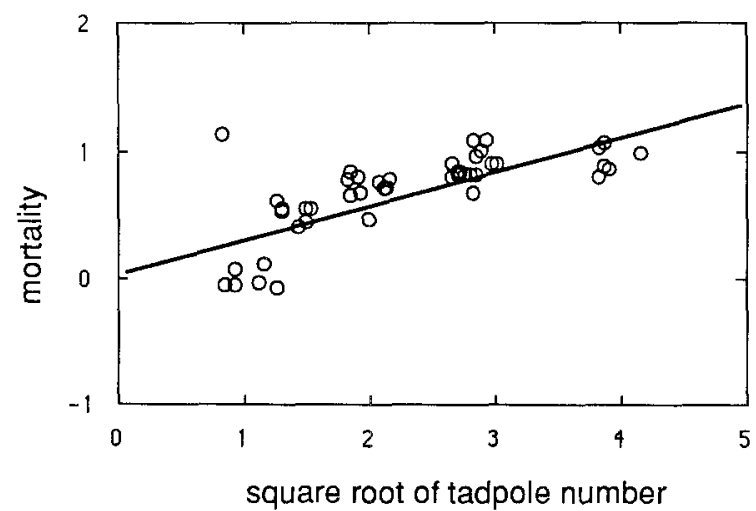

Fig. 4. Linear regression of average tadpole mortality on the square root of the number of tadpoles placed in the pool at the beginning of the experiment, at week 6. Since most points for a particular treatment level fell at the same place, a plotting function which randomly scatters identically placed points a small amount, so they can be visualized, was used 
Table 2. Week by week summary of the regression of tadpole mortality on the square root of the number of tadpoles placed in a pool at the beginning of the experiment, from week 1 through week $6 . N=$ sample size, $B=$ coefficient of the slope of the regression, $R^{2}=$ the amount of variance in mortality explained by the number of tadpoles in a pool, $P=$ significance level

\begin{tabular}{lllll}
\hline Week & $N$ & $B$ & $R^{2}$ & $P$ \\
\hline 1 & 50 & 0.017 & 0.013 & 0.417 \\
2 & 48 & 0.079 & 0.034 & 0.207 \\
3 & 41 & 0.221 & 0.066 & 0.100 \\
4 & 50 & 0.626 & 0.215 & 0.001 \\
6 & 42 & 0.283 & 0.940 & 0.000 \\
\hline
\end{tabular}

poles per pool. At week 6 , there was a significant positive relationship between the number of tadpoles in a pool and the square root of the degree of mortality in those pools (Fig. 4, $R^{2}=0.940, N=42, P=0.000$ ). Table 2 shows the regression coefficient, the amount of variance in mortality explained by tadpole number, the sample size and the level of significance for the regression of mortality on the square root of tadpole number from week 1 through week 6.

\section{Mortality vs growth rate}

The effect of the treatment (tadpole number at pool initiation) on mortality became increasingly significant with time (Table 2), whereas it's effect on growth became less significant with time (Fig. 1,2). It seems unlikely that this is coincidental. Within the higher tadpole number treatments, there were positive relationships between average growth rate and mortality. For example, there was a significant positive relationship between the degree of mortality and the $\log$ of average weight at week 4 in the 16 tadpole per pool treatments $\left(R^{2}=0.800, \mathrm{~N}=7\right.$, $p=0.000$ ).

The fact that the average growth rate was correlated with the amount of mortality within the high tadpole number treatments suggests that growth rate and mortality were causally related. Histograms of tadpole lengths for all tadpoles in pools started with 8 tadpoles at the beginning of the experiment, and after 2, 4 and 6 weeks (Fig. 5), show that the distribution of tadpole lengths at the start was approximately normal for all the pools, then became increasingly skewed toward a preponderance of smaller than average tadpoles, until week 6 , when it was slightly skewed in the other direction (towards large tadpoles).

Generally, in pools with 2 or more tadpoles, the distributions of tadpole size within the treatments became skewed over time, with 1 or 2 tadpoles growing rapidly, while the rest grew slowly or not at all. After 4 weeks the rate of mortality of the smaller tadpoles became very high (possibly because of competition or cannibalism by the larger tadpoles), and the few who had attained large size came to represent the average size in the high tadpole number treatments more and more. By week 6 , almost all the pools had only 1 large tadpole left in them, and these tadpoles were similar in size.
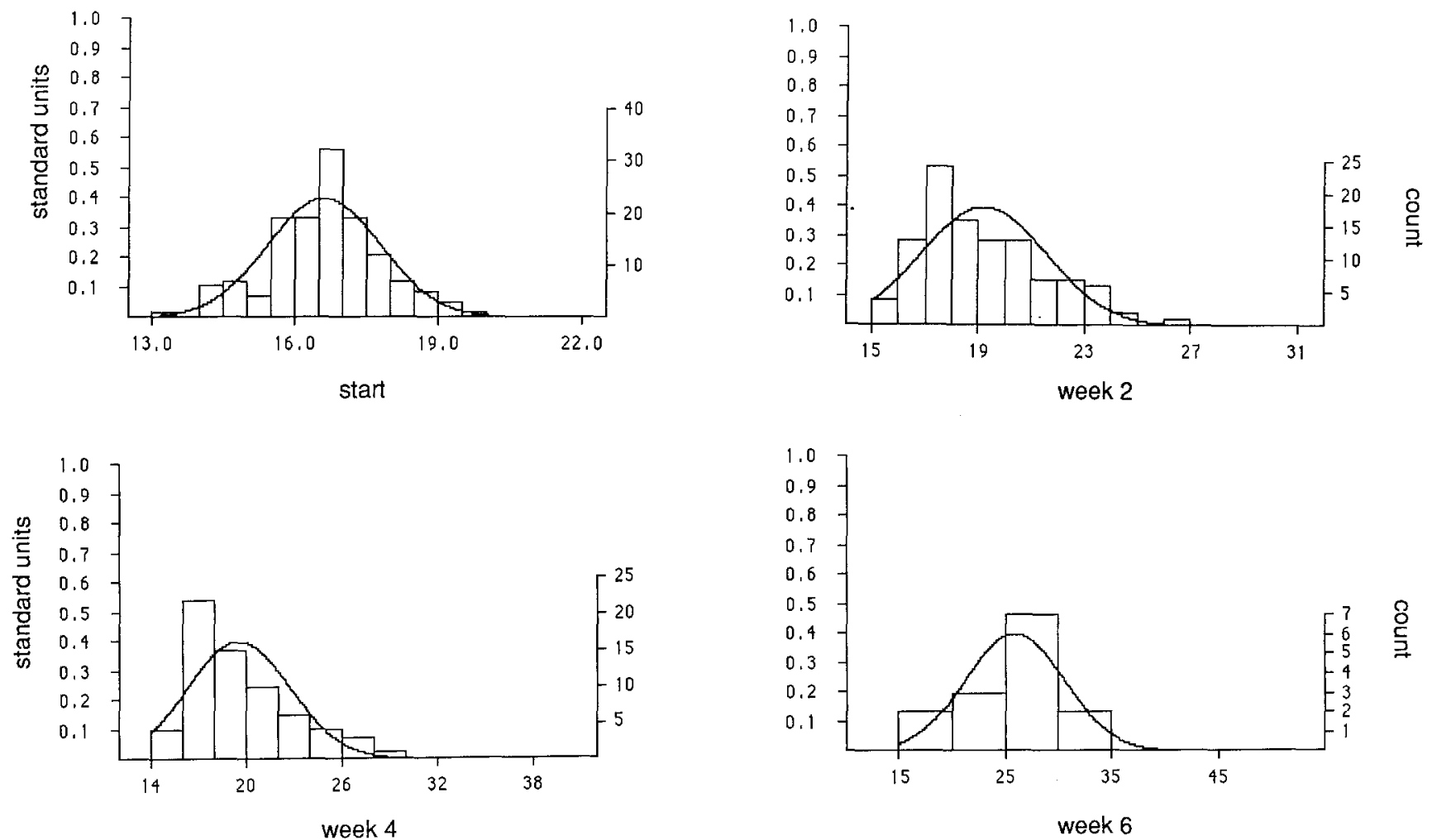

tadpole length

Fig. 5. Histograms of the distribution of tadpole length in all pools started with 8 tadpoles, at the beginning of the experiment, and at week 2,4 , and 6 . Count refers to the number of tadpoles in a size class, and Standard Units are standardized with respect to sample size, to allow comparison accross samples. A line representing the normal distribution is superimposed over each histogram 


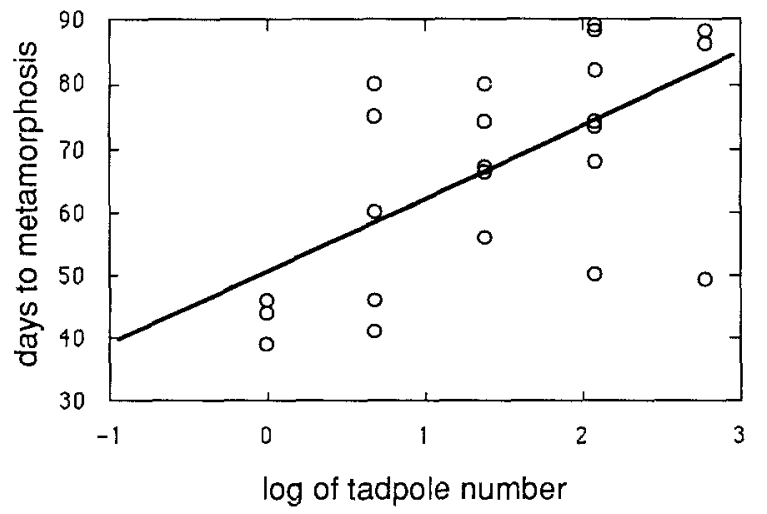

Fig. 6. Linear regression of the number of days until tadpoles reached metamorphosis on the $\log$ of the number of tadpoles in the pool at the start of the experiment

\section{Time to metamorphosis}

Time taken to reach metamorphosis averaged 66.1 days, and ranged from 39 to 89 days. There was a significant positive relationship between the number of tadpoles in a pool and the number of days it took any tadpoles from that pool to reach metamorphosis (Fig. $6, R^{2}=$ $0.321, N=23, P=0.003$ ). Tadpoles from 1 tadpole per pool treatments reached metamorphosis significantly earlier than tadpoles in pools with 2 tadpoles (MannWhitney $U, N=8, P=0.000$ ).

\section{Treehole comparisons}

The cohort with a lower number of tadpoles had a higher average growth rate and a lower mortality rate than the cohort with a higher number of tadpoles in 7 out of 8 of the treehole pools. High tadpole number cohorts had a median average growth rate $0.05 \mathrm{~g}$ (approximately equal to the average weight of a tadpole at deposition) lower, and a median average mortality rate 46 percent higher than that of the low tadpole number cohorts. One tailed tests were used because the results of the calabash pool experiment led me to expect that the cohorts with the higher numbers of tadpoles would have slower growth rates and higher mortality levels. A paired comparison of the average growth rates in the low versus high tadpole number cohorts, using a Wilcoxon test, showed that higher tadpole number cohorts had significantly higher levels of mortality $(P<0.005)$ and lower growth rates $(P<0.05)$.

\section{High food treatment}

There was a weak but significant negative relationship between tadpole number and growth in the high food experiment (Fig. 7, $R^{2}=0.319, N=15, P=0.028$ ). An analysis of covariance showed that the relationship between tadpole number and weight in the regular experiment $\left(R^{2}=0.472, N=47, P=0.000\right)$ was significantly more negative than that in the high food experiment (Fig. 7, food treatment by number $F=14.332, N=62$, $P=0.000)$.

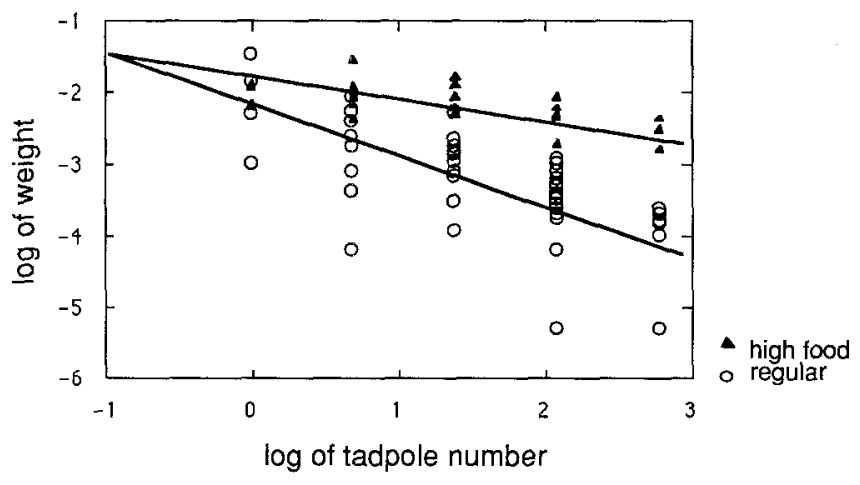

Fig. 7. Linear regressions of the $\log$ of average tadpole weight on the log of the number of tadpoles placed in the pool at the beginning of the experiment, for high food treatment pools and for regular pools, at week 1

\section{Dimorphic pairs treatment}

The smaller tadpoles had a higher rate of mortality than the larger tadpoles in the dimorphic pairs treatment (Large $=1 / 16$, Small $=11 / 16$ at week 6, Paired Sign Test, $N=16, P=0.006$ ). The growth rate of the smaller tadpoles in the dimorphic pairs treatment was significantly lower than the growth rate (as measured by total length) of the smaller tadpoles in the monomorphic pairs of the regular 2 tadpole per pool treatment after 1 week (Mann Whitney $U$ Test, $N=19, P=0.006$ ). There was insufficient data to compare growth rates after week 1, because of high mortality.

\section{Discussion}

Growth rate: interference or exploitative competition?

Why increasing tadpole number reduces average growth rate is unknown, but the high food experiment suggests that both exploitative competition for available nutrients and aggressive interference effects play a part. The negative relationship between tadpole number and growth rate in the high food experiment, indicates that, even when the tadpoles have nutrients abundantly supplied to them, there is still a negative effect of tadpole number on growth rate. This suggests that direct aggressive interactions may be lowering growth rates in some individuals (possibly through an effect of stress on hormone levels), or tadpoles may be releasing growth inhibiting substances (Rose 1960). On the other hand, the analysis of covariance (pg. 8) showed that the relationship between tadpole number and growth rate at week 1 was significantly less negative in the high food experiment, suggesting that there is an effect of exploitative competition for available nutrients in the regular calabash pools.

\section{Mortality: starvation or aggression?}

Whether mortality was higher in pools with more tadpoles because of lack of food, or because of increased aggressive interactions, was not certain, but a variety 
of evidence suggests that tadpoles were killed by other tadpoles, rather than simply starving to death. Tadpoles were frequently observed cannibalizing dead tadpoles, and attacking and biting other (live) tadpoles. 2 tadpoles of another species (Physalaemus pustulosus) were killed and torn apart within half an hour of being placed in a pool with several $D$. auratus tadpoles. Tadpoles in the pools had pieces missing from the tail, apparently from attacks by other tadpoles.

An experiment identical to the main tadpole pool experiment, but in plastic cups with low detritus levels, demonstrated that single tadpoles can survive for months with almost no detritus and no other source of food. Hence, it seems unlikely that the other tadpoles in the pools died of starvation alone. It is, however, possible that those tadpoles who were getting the least amount of food were the most likely to die from the attacks of other tadpoles. Mortality in larger pools with more nutrients is probably not as high as it was in the calabash experiments, where there was usually only one surviving tadpole. For example, I have seen several tadpoles that had almost reached metamorphosis in single large treehole pools on Taboga.

\section{The cost of polygyny}

The observations reported here showing that males frequently deposit more than one tadpole in the same pool, combined with observations showing that males frequently attempt to be polygynous (Summers 1989, unpublished observations), indicate that females face a real risk of having their mates deposit tadpoles from other females in the same pools as their own tadpoles. All multiple depositions observed in this study were within the minimum period required for tadpoles to reach metamorphosis (39 days), so that tadpoles were interacting with previously deposited tadpoles. The data from the calabash pool and treehole experiments indicating that increasing the number of tadpoles placed in a pool reduces the early average growth rate, increases the amount of mortality within the pool, and increases the time taken to reach metamorphosis, suggest that females are likely to suffer a cost, in terms of reduced offspring growth and survivorship, when their mates deposit tadpoles from other females in pools with their own offspring.

These are likely to be biologically significant costs to the reproductive success of both parents. Because females gain no offsetting benefits from their mate's care of other female's offspring, they suffer a net cost from male polygyny, and hence should attempt to prevent such polygyny if possible.

The range of tadpoles found in pools in the field (treeholes and calabashes) varied from 1 to 14 . These are likely to be underestimates of the number deposited in a pool because of tadpole mortality, which was high in experiments with both treehole and calabash pools (see Results). In pools that are easily accessible in areas of high population density, deposition rates are likely to be high. For example, in plastic cups placed in areas of high population density, tadpole depositions averaged
2 or 3 per day. On the other hand, natural pools located away from centers of population density often had only one or a few tadpoles when searched (the per day deposition rates for these pools are not known, because they were not tadpole collection pools). I believe that the range of tadpoles per pool used in the experiments was representative of that found in nature. The 16 tadpole per pool treatment may be somewhat high, but the removal of that treatment does not reduce the significance of the effect of tadpole number on growth rate or survivorship found in the experiments. Thus, the costs demonstrated are likely to be biologically significant under natural circumstances.

This is the first experimental study of any species (to my knowledge) explicitly designed to test the hypothesis that females suffer a cost (in terms of reduced offspring growth or survivorship) as a result of their mate's polygyny. The results of this study, combined with previous evidence of prolonged mate guarding by females in D. auratus (Summers 1989), suggest that females attempt to prevent their mates from being polygynous in order to avoid a cost to their offspring's fitness.

The idea that females aggressively guard their mates in order to avoid suffering a cost from male polygyny has been discussed in the literature (e.g. Blaffer Hrdy and Williams 1983), but unambiguous evidence of either female competition to reduce a mate's degree of polygyny, or of a cost to females from a reduction of the quality of paternal care, due to polygyny, is rare. There are several possible reasons that these phenomena have not been observed frequently. In many species, particularly in mammals, the effect of paternal care on offspring growth and survivorship is not easily quantifiable and not amenable to experimentation (e.g baboons, Wasser 1983). In many polygynous species males may attempt to prevent female mate guarding, by interference in female-female aggression or by attempting to segregate their mates. In some birds in which female competition for mates has been studied, males invest in only 1 clutch at a time, females can produce offspring more rapidly than males can care for them, and there is higher variance in female than in male reproductive success (e.g. Jacanas, Jenni 1974; Spotted Sandpipers, Oring 1986). This leads to what Trivers (1972) called sex role reversal, and the effect of this type of selection on male and female mating strategies is different from that exerted by uncertainty of parental investment (Summers 1989). It is also possible that previous researchers have tended to ignore or discount the occurrence of female-female competition for mates when male polygyny is common, because of an historical emphasis on male-male competition in such species (Wasser and Waterhouse 1983).

A likely example of another species in which femalefemale aggression occurs in response to a cost of male polygyny is the red-winged blackbird (Agelaius phoeniceus). Yasukawa and Searcy (1982) performed dummy presentation experiments which showed that females preferentially attack female dummies in copulation solicitation posture in the female's mate's territory, suggesting that female-female aggression is a form of mate guarding designed to keep the male from caring for the 
offspring of other females. Patterson (1979) showed that paternal care (nestling provisioning) has a significantly positive effect on offspring growth and survivorship. In some populations, females are believed to care only for the offspring of their first mate (Yasukawa and Searcy 1982), but Whittingham (1989) has demonstrated experimentally that males will preferentially provision the offspring of larger clutches in a population in Michigan, and so it is probable that females face a potential reduction in offspring growth and survivorship when their mates mate polygynously. A cost of polygyny may also be responsible for female aggression in Pied Flycatchers (Ficedula hypoleuca; Breiehagen and Slagsvold 1988).

\section{Female fitness and the timing of tadpole deposition}

The results of the size treatment experiment (p. 8) suggest that small tadpoles have a higher risk of mortality than large tadpoles, and have slower growth rates relative to tadpoles in pools with another tadpole of similar size. From a male's perspective, placing a new tadpole in a pool where he has put one previously may increase the number of his offspring coming out of the pool, if sufficient nutrients are available, or provide food for the first if nutrients are scarce. Females, then, should attempt to ensure that their tadpoles have a higher probability of being the first sibling that a male places in a pool, if possible.

Some observations of female guarding behavior on Taboga Island suggest that some females tend to guard more intensely for a week or two before mating with a male. For example, in 1985, a female was seen in a male's area regularly for several weeks, and was observed to court with the male repeatedly and attack another female who attempted to court with the male. After 2 weeks, the guarding female mated with the male, and then was not observed in the area for 2 weeks. Another female (also in 1985), was regularly seen courting the male in her area for 10 days, and attacked two other females who attempted to court with the male during that time. She then abruptly stopped courting with the male, and although she remained in the area, she did not attack other females courting the male, even when they passed within a few inches of her. Unfortunately, it is not known if this dramatic change in behavior occurred near the time that the guarding female mated with the male, because this event was not observed. However, some females guarded the male in their area consistently throughout the observations ( 10 weeks).

Guarding males more intensely from a few weeks prior to a few days after mating would be most likely to benefit females if: 1) tadpole deposition is clumped in time and punctuated by long (e.g 2 week) intermissions. This seems likely on Taboga Island, where there are long dry spells even during the rainy season, and where more males carry tadpoles during wet weather (personal observations). 2) Males are likely to look for new pools between pulses of deposition, so that they may be putting at least some of the tadpoles from a new pulse into new pools. Testing the hypothesis that some females guard males in a way that maximizes the probability that their tadpoles will be the first ones deposited in newly discovered pools would require more extensive field observations on female guarding behaviour, rates of oviposition, and male tadpole deposition patterns.

Acknowledgements. I would like to thank A. Stanley Rand, Richard D. Alexander, Nancy Knowlton and Arnold G. Kluge for advice and support throughout the research and writing involved in this paper. The paper also benefitted from comments from Earl Werner, Richard Connor, and Beverly Strassmann. The field work was assisted by the staff of the Smithsonian Tropical Research Institute, and by research permits from RENARE in the Republic of Panama. The research was made possible by a pre-doctoral fellowship from the Smithsonian Institution. I would especially like to thank my assistant, Michael Barry, for perserverance above and beyond the call of duty.

\section{References}

Alexander RD, Borgia $G$ (1979) On the origin and basis of the male-female phenomena. In: Blum MS, Blum NA (eds) Sexual selection and reproductive competition in insects. Academic Press, New York, pp 417-440

Bateman AJ (1948) Intra-sexual selection in Drosophila. Heredity $2: 349-368$

Blaffer Hrdy S, Williams GC (1983) Behavioral biology and the double standard. In: Wasser SK (ed) Social behavior of female vertebrates. New York, Academic Press, pp 3-17

Breiehagen T, Slagsvold T (1988) Male polyterritoriality and female-female aggression in Pied Flycatchers Ficedula hypoleuca. Anim Behav 36:604-605

Jenni DA (1974) Evolution of polyandry in birds. Am Zool $14: 129-44$

Oring LW (1986) Avian polyandry: a review. In: RF Johnson (ed) Current ornithology, vol 3. Plenum Press, New York, pp 309 351

Patterson CB (1979) Relative parental investment in the red-winged blackbird. Ph.D. Thesis, Indiana University

Rose SM (1960) A feedback mechanism of growth control in tadpoles. Ecology $41: 188-199$

Summers K (1989) Sexual selection and intra-female competition in the green poison-dart frog, Dendrobates auratus. Anim Behav 37:797-805

Thornhill R., Alcock J (1983) The evolution of insect mating systems. Harvard University Press, Cambridge, MA

Trivers RL (1972) Parental investment and sexual selection. In: B Campbell (ed) Sexual selection and the descent of man, 18711971. Aldine Press, Chicago, pp 136-179

Wasser SK (1983) Reproductive competition and cooperation among female yellow baboons. In: SK Wasser (ed) Social behavior of female vertebrates. Academic Press, New York, pp $350-390$

Wasser SK, Waterhouse ML (1983) The establishment and maintenance of sex biases. In: SK Wasser (ed) Social behavior of female vertebrates. Academic Press, New York, pp 19-35

Whittingham LA (1989) An experimental study of paternal behavior in red-winged blackbirds. Behav Ecol Sociobiol 25:73-80

Wilkinson L (1987) Systat: the system for statistics. Systat, Evanston, IL

Williams GC (1966) Adaptation and natural selection. Princeton University Press, Princeton

Wittenberger JF (1979) The evolution of mating systems in birds and mammals. In: P Marler, JG Vandenburg (eds) Handbook of behavioral neurobiology, vol 3, Social behavior and communication. Plenum Press, New York, pp 271-349

Yasukawa K, Searcy WA (1982) Aggression in female red-winged blackbirds: a strategy to ensure male parental investment. Behav Ecol Sociobiol 11:13-17 